\title{
A new theory to explain geometrical illusions produced by crossing lines
}

CHUNG CHIANg

POLYTECHNIC INSTITUTE OF BROOKLYN

Due to the diffraction of light and other optical distortions of the eye, the image of an object is not exactly the same as the object. When two objects are close enough, their two images overlap so as to form one image, located at a position somewhere between the two original images. This fact is used to explain illusions produced by the crossing of lines, including Poggendorff's, Zollner's, Hering's, Wundt's, the Müller-Lyer and other illusions of this class.

Since geometrical lllusions were first reported in the literature, various theories have been proposed to explatn them. Often, a theory as advanced explains one case but fails to explain the other cases.

Oster (1966) has pointed out that Poggendorff's illusion may be due to the inability of the eye to resolve the intersection, for reasons not explained. In this paper, a theory has been proposed which uses the diffraction image to explain Poggendorff's illusion, and then uses Poggendorff's illusion to explain all geometrical illusions produced by crossing lines.

When two lines, $\mathrm{AA}^{\prime}$ and $\mathrm{BB}^{\prime}$, cross each other as shown in Fig. 1, the lines appear to be broken. If one of the lines such as $A^{\prime}$ is wider than $B^{\prime}$, then the fact that $\mathrm{BB}^{\prime}$ appears to be broken is more obvlous. If the crossing angle is small, this phenomenon is also more obvious. If the crossing angle is $90^{\circ}$,

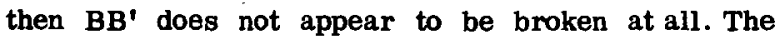
phenomenon, that BB' appears 'broken, is known as Poggendorf's lllusion.

When one views an object, the light of the object passes through the eye lens and forms an optical image on the retina. However, due to the aberrations of the lens and the diffraction of the light, the optical image will never be exactly the same as the object (Jenkins \& White, 1957, pp. 288-309).

When two objects are well separated, the eye can see those objects as two separated objects. Howerer, when two objects are close enough together, the eye no longer sees two separated objects, but only one combined object. Due to diffraction, the distribution of light intensity of the image on the retina does not exactly correspond to the shape of the object. If the object has a sharp edge, the image may have a blurred edge, and the distribution of light intensity in the image falls off gradually instead of sharply at the edge. In Fig. 2, the diffraction images of two slits are shown. The intensity distribution is plotted vertically, and the distance is plotted hori-

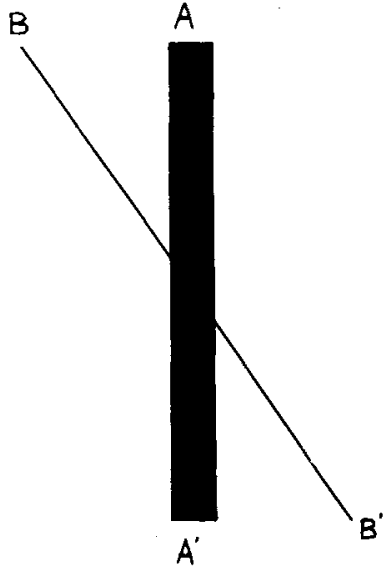

Fig. 1. Poggendorf's illusion. The thin line appears to be broken.

zontally. The dotted and the light curves are the diffraction images of the two slits, respectively, and the heavy line is the resultant shape of the final image, which is obtained by adding the intensity of the separated curves (dotted and light curves). In Fig. 2a, one can easily see the two maxima of the

(a)

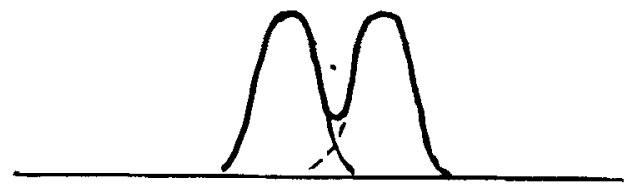

(b)

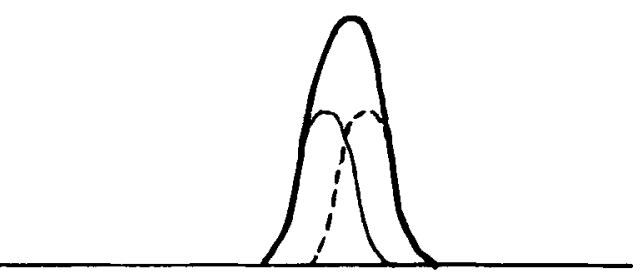

Fig. 2. Diffraction images of two slit sources. (a) One can see two maxima of intensity, corresponding to two objects. (b) One can see one maximum; thus two objects seem merged to form one image. 


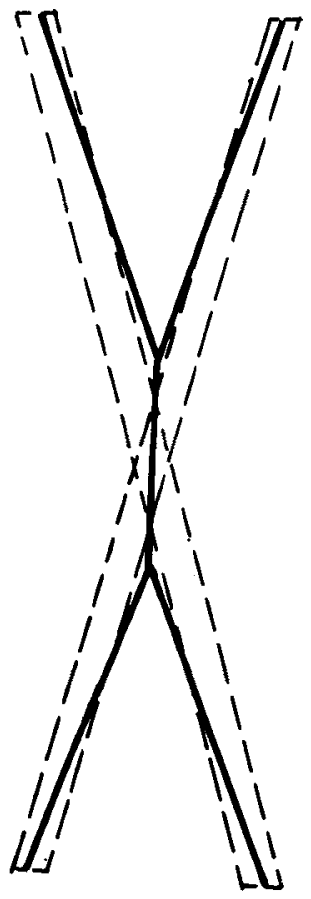

Fig. 3. The distribution of the maximum intensity of the two crossing slit sources (heavy line). The dashed line encloses the shape of the original slits. Note that it is exaggerated.

intensity; therefore, one sees two slits. As the two slits move gradually closer to each other, the resultant curve changes, untll a stage is reached in which only one maximum of intensity exists. The two slits can no longer be seen as two slits, and it appears that there is only one source, even though one knows that there are actually two sources.

One notes that the image of the object has a maximum of intensity at the center, which roughly corresponds to the location of the object. This maximum of intensity has a certain width, which roughly corresponds to the width of the object. However, the intensity of light falls off gradually at the edge of the image, unlike the sharp edge of the object. In addition, when two objects move closer together so that only one object is seen, the final pattern has only one maximum instead of two maxima, and the position of the maximum of the resultant pattern is at the position in the middle between the two maxima of the component curves. In other words, instead of seeing two objects, one sees a single object which is located at the position somewhere between the two original objects.

Let us now return to Poggendorff's illusion. For two crossing lines $\mathrm{AA}^{\prime}$ and $\mathrm{BB}^{\prime}$ (see Fig. 1), Points $A$ and $B$ are well separated, so the eye has no problem in seeing two separated lines. As the two lines gradually converge, it is more and more difficult for the eye to see them as two separated lines. Finally, at a point very close to the crossing point (just before the crossing point is reached), the eye is not able to distinguish the two lines; the two lines seem to have converged to one line. Furthermore, the position of the maximum intensity is shifted to a point somewhere between the two original lines. In Fig. 3, the position of the resultant maximum intensity is shown with the heavy line, and the original lines $\mathrm{AA}^{\prime}$ and $\mathrm{BB}^{\prime}$ are enclosed with dashed lines. One sees that the heavy lines rotate through an angle from the original direction. Since the heavy lines are the main impression one gets in the retina, even though the whole area inside the dashed lines can be seen, it appears that the lines are broken, and the lines rotate through an angle.

If the two lines cross each other with a smaller angle, then the two lines begin to converge at a position farther away from the crossing point. If the two lines cross each other with a larger angle, then the two lines begin to converge at a position closer to the crossing point. Therefore, the lines appear to be broken more obviously with a smaller degree of crossing angle. When line $A^{\prime}$ ' is wider than $\mathrm{BB}^{\prime}$ (see Fig. 1), the intensity distribution of line $\mathrm{AA}^{\prime}$ is not changed very much by the thin $\mathrm{BB}^{\prime}$ line, but the intensity distribution of the thin line, $\mathrm{BB}^{\prime}$, is changed considerably by the wide line, $\mathrm{AA}^{\prime}$. Therefore line $\mathrm{AA}^{\prime}$ appears to be a straight line, but line $\mathrm{BB}^{\prime}$ appears to be broken and rotates through an angle.

This account explains the Poggendorff illusion. Once Poggendorff's illusion is explained, it is easy to explain other illusions produced by crossing lines. If there are many crossing points, then there are

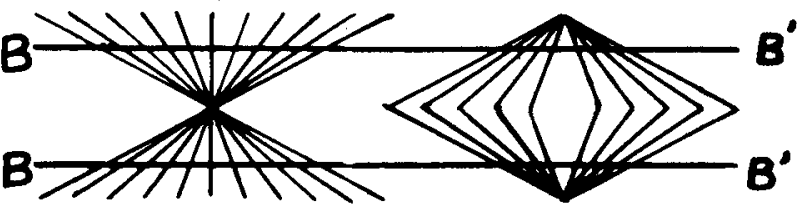

\section{(a)}

(b)

Fig. 5. The line is curved away from the radial point. (a) Hering's illusion. (b) Wundt's illusion. 


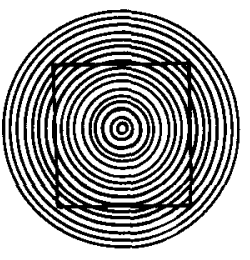

(a)

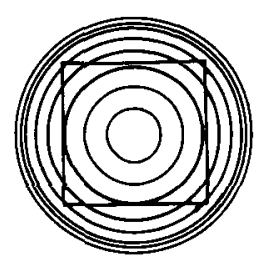

(b)

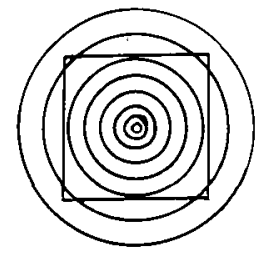

(C)
Fig. 6. The square appears bowed inward regardless of the distance between any two successive circles.

many Poggendorff's illusions. When many Poggendorff's illusions are correlated, a new phenomenon appears. The following are some examples.

If line $\mathrm{BB}^{\prime}$ is crossed by many parallel lines (see Fig. 4), then for every crossing point, Poggendorff's illusion operates. Since the angles formed by line $\mathrm{BB}^{\prime}$ with all the parallel lines are the same, the Poggendorff's illusions all operate to the same degree. Therefore the $\mathrm{BB}^{\prime}$ line still appears a straight line, but rotates through an angle. Reverse the direction of those parallel lines, then the direction of the rotation of the $\mathrm{BB}^{\prime}$ line is also reversed. This illusion is usually known as Zollner's illusion.

If line $\mathrm{BB}^{\prime}$ is crossed by radial lines (see Fig. 5), then the crossing angles are different for every crossing point; thus, Poggendorff's Illusions operate to different degrees at different crossing points. Thus, line $B^{\prime}$ ' appears to be curved. From examining the Poggendorff's illusion, the nature of the curve can be determined by finding out in which direction the line should rotate. In this way, one finds that line $\mathrm{BB}^{\prime}$ should curve away from the radial point, and indeed the line appears to do so. Figure 5a is known as Hering's illusion and Fig. $5 \mathrm{~b}$ as Wundt's illusion.

If a square is put within the background of circles,
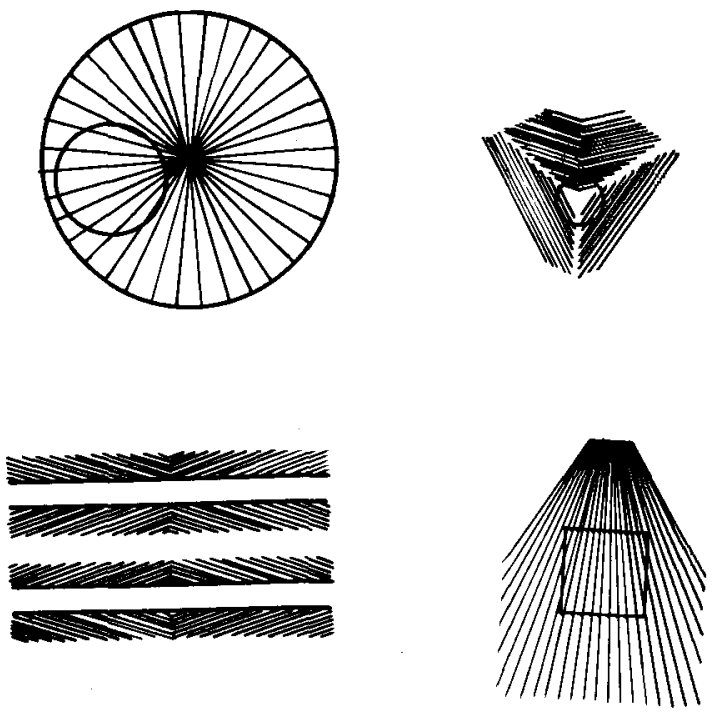

Fig. 8. Some other examples that can be explained by the Poggendorff's illusion.

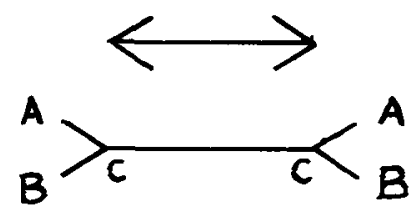

Fig. 7. Müller-Lyer illusion.

the square appears bowed in, regardless of the distance between any two successive circles (see Fig. 6). As pointed out by Green and Stacey (1966), Gregory's theory will predict that the square looks bowed in the opposite direction in Figs. $6 \mathrm{~b}$ and $6 \mathrm{c}$. This is not the case; therefore, the theory fails in this instance. However, when one examines the squares in these cases on the principle of Poggendorff's illusion, one finds that the square should look bowed inward. Since only the angle of the crossing points affects Poggendorff's illusion, and the angles of the crossing points in these cases are all similar, the squares appear bowed inward in the same direction regardless of the distance between any two successive circles.

It should be noted that the number of crossing points in unit length is very important in the illusion. If the number of crossing points in the unit length is large, the illusion is stronger (Oster, 1966b).

The Müller-Lyer illusion can also be explained by the theory of diffraction image. The two horizontal lines in Fig. 7 are actually equal, but due to diffraction of the light, lines $A C$ and $B C$ converge before they actually reach the crossing point in the image. This small addition of length makes it appear that the lower figure has a longer horizontal line.

Many other illusions of this type can be explained in the same way. Figure 8 includes some additional examples.

In conclusion, one may say that when two objects are close enough, the two images of the objects overlap to form one image, which is located somewhere between the two original images. This fact is used to explain Poggendorff's lllusion, which is in turn used to explain other illusions produced by crossing lines. It is pointed out that the crossing angle and the number of crossing points per unit length are important factors in these effects.

\section{References}

Green, R. T., \& Stacey, B. G. Misapplication of the misapplied constancy hypothesis. Life Sci., 1966. 5, 1871-1880.

Jenkins, F. A., \& White, H. E. Fundamentals of optics, New York: McGraw-Hill, 1957. Pp. 288-309.

Oster, G. A similar situation has been mentioned in Optical Art. Appl. Opt., 1965, 4, 1359-1368.

Oster, G. The Scicnce of Moire Patterns. Barrington, New Jersey: Edmound Scientific Co., 1966. Pp. 22-23.

(Accepted for publication November 6, 1967.) 